\title{
Understory structure in two successional stages of a Semi-deciduous Seasonal Forest remnant of Southern Brazil
}

\author{
Geraldo Ceni Coelho ${ }^{1,3}$, Marivana da Silva Rigo ${ }^{2}$, Juliana Boniatti Libardoni², \\ Rosemeri de Oliveira ${ }^{2}$ \& Glaci Benvenuti-Ferreira ${ }^{2}$ \\ ${ }^{1}$ Departamento de Biologia e Química, UNIJUÍ, MEC/Sesu/PET fellow, \\ Universidade Federal da Fronteira Sul - UFFS, Av. Presidente Getúlio Vargas, 609N, CEP 89812-000, \\ Edifício Engemede $2^{\circ}$ andar, Centro, Chapecó, SC, Brazil \\ ${ }^{2}$ Undergraduate of Ciências Biológicas, \\ Universidade Regional do Noroeste do Estado do Rio Grande do Sul - UNIJUÍ, Rua do Comércio, 3000, \\ CEP 98700-000, Universitário, Ijuí, RS, Brazil \\ ${ }^{3}$ Corresponding author: Geraldo Ceni Coelho, e-mail: cenicoelho@gmail.com
}

COELHO, G.C., RIGO, M.S., LIBARDONI, J.B., OLIVEIRA, R. \& BENVENUTI-FERREIRA, G. Understory structure in two successional stages of Semi-deciduous Seasonal Forest remnant of Southern Brazil. Biota Neotrop. 11(3): http://www.biotaneotropica.org.br/v11n3/en/abstract?article+bn01011032011

\begin{abstract}
The knowledge of structure and composition of forest strata contributes to understanding the conservation status and dynamics of plant communities. The study of forest understory is important to the knowledge of the succession process. The Seasonal Subtropical Forest remnant named Mato do Silva (27 $55^{\prime} 26^{\prime}$ ' S and 53 53' $15^{\prime \prime}$ ' W) was studied aiming to describe the structure of understory in the late successional forest (LF) and in the young secondary forest (SF), an 11 years old post-agricultural site with canopy dominance of Ateleia glazioveana Baill. The phytosociological survey was carried out through the point quarter method and included individuals of tree and shrub species from 0.5 to $3.0 \mathrm{~m}$ of height. The sampling consisted of 384 individuals in LF (67 species) and 284 individuals in SF (20 species). A total of 79 species were observed, and only eight species occurred in both areas. The specific diversity (H') was 3.45 and 2.42 nats in the understory of LF and SF, respectively. The Pielou's evenness index (J) was 0.85 to LF and 0.80 to SF, respectively. Cupania vernalis Camb. and Psychotria leiocarpa Müll. Arg. were the prominent species in the LF while A. glazioveana and Solanum mauritianum Scop. were outstanding in the SF understory. Shade-tolerant and late secondary tree species occurred in large proportion in the LF forest, while pioneers and early secondary tree species predominated in the SF. In addition, certain dominant species in the understory of the SF were absent in the LF understory, such as A. glazioveana, Solanum compressum L. B. Sm. \& Downs, Helietta apiculata Bentham and Gledtisia amorphoides (Griseb.) Taubert, which indicates that such species are associated to early stages of forest succession. G. amorphoides is considered an endangered species, and the early successional stages could be necessary for conservation of this species. A decrease in the $\mathrm{pH}$ and mineral nutrient levels in the soil was linked to the successional process, indicating the transference of nutrients to the aboveground biomass.

Keywords: biodiversity conservation, biological nitrogen fixation, secondary succession, atlantic forest of Uruguai river basin, subtropical forest.
\end{abstract}

COELHO, G.C., RIGO, M.S., LIBARDONI, J.B., OLIVEIRA, R. \& BENVENUTI-FERREIRA, G. Estrutura do sub-bosque em duas fases sucessionais de um fragmento de Floresta Estacional Semi-decidual do sul do Brasil. Biota Neotrop. 11(3): http://www.biotaneotropica.org.br/v11n3/pt/abstract?article+bn01011032011

Resumo: O conhecimento da estrutura e composição dos estratos florestais contribui para a compreensão do estado de conservação e dinâmica das comunidades vegetais. O estudo do estrato inferior florestal é importante para o conhecimento sobre os processos de sucessão. O remanescente de Floresta Subtropical Estacional denominado Mato do Silva $\left(27^{\circ} 55^{\prime} 26^{\prime}\right.$ ' S e 53 53' $15^{\prime}$ ' O) foi estudado objetivando descrever a estrutura do estrato arbustivo na floresta em estádio avançado de sucessão e na floresta secundária, sendo esta formada em uma antiga área agrícola abandonada há 11 anos, e que possui estrato superior dominado por timbó (Ateleia glazioveana Baill.). A amostragem fitossociológica foi realizada através do método de quadrantes e incluiu indivíduos de espécies lenhosas arbustivas e arbóreas entre 0,5 e $3 \mathrm{~m}$ de altura. Foram amostrados 384 indivíduos na área de floresta avançada (67 espécies) e 284 na área de floresta secundária (20 espécies). Foram observadas 79 espécies no total, e somente oito ocorreram em ambas as áreas. A diversidade específica ( $\left.H^{\prime}\right)$ foi de 3,45 e 2,42 nats, respectivamente. $\mathrm{O}$ índice $\mathrm{J}$ de equitabilidade foi 0,85 para LF e 0,80 para SF, respectivamente. Na área de floresta estacional avançada, destacaram-se Cupania vernalis Camb. e Psychotria leiocarpa Müll. Arg., enquanto que na floresta secundária A. glazioveana e Solanum mauritianum Scop. As espécies esciófilas ou secundárias tardias foram dominantes no estrato inferior da floresta estacional avançada, enquanto que na floresta secundária predominaram as espécies arbóreas pioneiras e secundárias iniciais. Além disso, certas espécies dominantes na floresta secundária estão ausentes no estrato inferior da floresta avançada, tais como A. glazioveana, Solanum compressum L. B. Sm. 
\& Downs, Helietta apiculata Benth. e Gledtisia amorphoides (Griseb.) Taubert, o que indica que estas espécies estão associadas a estádios iniciais de sucessão florestal. G. amorphoides é considerada em perigo de extinção, e os estádios sucessionais iniciais podem ser necessários para a conservação desta espécie. Observou-se uma redução do pH e concentração de nutrientes minerais associada ao processo sucessional, o que indica transferência de nutrientes para a biomassa aérea.

Palavras-chave: conservação da biodiversidade, fixação biológica de nitrogênio, sucessão secundária, Floresta Atlântica da Bacia do Rio Uruguai, Floresta Subtropical.

\section{Introduction}

The Northwest of the State of Rio Grande do Sul, between Turvo and Ijuí River basins, corresponds to a region originally dominated by the Subtropical Seasonal Forest, which presents a high fragmentation (Granell-Pérez et al. 1999, Schenkel et al. 2003, FUNDAÇÃO SOS MATA ATLÂNTICA \& INPE 2002) and is reduced to only $6 \%$ of its original cover of $1,000,000 \mathrm{~km}^{2}$ among Brazil, Argentine and Paraguay (Burkart \& Fernández 2002). The forest resources exploitation and the rise of crop cultivation have conducted to a drastic decrease in the wild plant communities at that ecoregion, undermining biodiversity maintenance. On this way, the knowledge of communities and ecosystems dynamics in patch landscapes contributes to their conservation, since these remnants include rare and endangered animals and plants (Nascimento et al. 2001). The understanding of species composition and functional groups in the early sucessional stages could contribute to ecological restoration, as the species choice is crucial for recovering planning and management success.

The tree strata replacement and the dynamics of forest diversity depend on the balance of mortality and recruitment in different stages of plant community dynamics (Clark et al. 1999, Wills et al. 2006) especially in the secondary succession process (Kennard 2002). Due to the viability decay of crop cultivation in tropical and subtropical regions, the secondary succession became a main process in several landscapes. In this context, the diversity maintained by secondary formations in different scales contributes decisively to the structure of the ecosystems and the landscapes in the Neotropics (Marín-Spiotta et al. 2007).

Secondary succession does not only imply in qualitative and quantitative changes of floristic composition, but also includes changes in abiotic factors such as soil and available light. The availability and cycling of nutrients could change along the succession (Compton et al. 2007, Davidson et al. 2007), consequently floristic composition could be related to nutrient limitations. For instance, after crop abandonment nitrogen $(\mathrm{N})$ mineralization can fall in the first years, increasing gradually (Vitousek et al. 1989). If the $\mathrm{N}$ availability is a limiting factor in early secondary succession following farming abandonment (Davidson et al. 2007), the biological nitrogen fixation and the leguminous tree species can be favored. Acidity, exchangeable bases and $\mathrm{Al}$, and effective cation-exchange capacity can also be modified along the succession, especially in the first decades (Hughes et al. 1999, Bautista-Cruz \& del Castillo 2005).

Dispersions modes could also be modified in the secondary succession. The zoochoric species and individuals dominate the tropical and subtropical forests (Howe \& Smallwood 1982, Budke et al. 2005), including the Seasonal Forests from Southern Brazil (Giehl et al. 2007, Ruchel et al. 2007). On the other hand, zoochory is greater among the late successional species (Benvenuti-Ferreira \& Coelho 2009), suggesting a shift in the dispersion modes during the secondary succession.

This paper aimed to compare understory stratum of two different successional stages in a Semi-deciduous Seasonal Forest of Southern Brazil in order to measure the plant diversity in a small fragment, and to evaluate the contribution of secondary succession to the local diversity. On this way, two successional stages were compared in terms of floristic and phytossociological data, ecological groups of dispersion and regeneration. In addition, the physicochemical soil properties of the different successional stages were compared, and the proportion of leguminous trees with biological nitrogen fixation was counted, seeking to identify an association between plant community structure and nutrient cycling.

\section{Materials and Methods}

The study site $\left(27^{\circ} 55^{\prime} 11^{\prime \prime} \mathrm{S}\right.$ and $\left.53^{\circ} 52^{\prime} 40^{\prime \prime} \mathrm{W}\right)$ is located in Chiapetta municipality, State of Rio Grande do Sul, Brazil, (Figure 1). The climate is SL PU perhumid subtropical with average annual temperature of $19{ }^{\circ} \mathrm{C}$ and annual rainfall of 1,800 mm (Maluf 2000). The altitude ranges between 400 and $472 \mathrm{~m}$ above mean sea level. The forest remnant has 240 ha of late successional Semi-deciduous Seasonal Forest and around 50 ha of areas of secondary shrubby or forest vegetation and marsh areas (Figure 1). It is considered here late successional the forest without known perturbations in the last 30 years according to local informants, and to which structure of tree strata was described previously (Benvenuti-Ferreira \& Coelho 2009). That late successional forest is similar to other non-riparian Seasonal Forests in the region (Vasconcellos et al. 1992, Ruchel et al. 2007). The young secondary forest corresponds to an abandoned cropland during 11 years, whose canopy is almost exclusively dominated by the timbó (Ateleia glazioveana Baill., Fabaceae), and whose higher individuals did not surpassed $15 \mathrm{~m}$ height.

The physiognomy of early secondary shrubby vegetation was dominated by A. glazioveana or by a combination of Baccharis dracunculifolia DC and Poaceae species (Saccharum villosum Steud., Schizachyrium microstachyum (Ham.) Roseng. et al., and Phalaris angusta Nees ex Trinius, among others).

The geology of the region belongs to the Serra Geral domain, with basaltic rocks derived from volcanisms of Jurassic and Cretaceous, which produced several lava overflows (Rambo 1956). The prevalent soil is Oxisols (United... 1999) with low nutrients levels and acid pH. The soil samples were collected on each site in the 3-10 cm depth interval, through the sum of ten subsamples of nearly $100 \mathrm{~g}$ each one, randomly distributed in an area of 1 ha into the phytossociological sample area. The soil subsamples were mixed and homogenized prior to the analysis. The physicochemical soil data were provided by UNIJUÍ Soil Laboratory, and obtained under the recommendations of Tedesco et al. (1995). The soil samples were collected in May 2009, October 2009 and March 2010, in the young secondary forest, in the late successional forest and also in the shrubby secondary vegetation adjacent to the young secondary forest, aiming to evaluate physicochemical changes related to secondary succession. It is assumed here that this vegetation is likely a more early successional stage than the composition dominated by A. glazioveana. The physicochemical data from the different areas were compared through a one-way ANOVA and a pairwise Tukey test $(p<0.05)$.

The phytosociological survey was carried out in the late and young successional forest. The identification and sampling of ligneous plants with height between 0.5 and $3.0 \mathrm{~m}$ was carried out 
from September 2006 to October 2007, through the Point Quarter Method (Cottam \& Curtis 1956) with points every $10 \mathrm{~m}$, totalizing 96 and 71 points in the late successional forest and young secondary forest, respectively. All diameters were measured $20 \mathrm{~cm}$ from the ground using a pachymeter.

Data from both understories were compared with the tree strata in the late successional forest previously published (Benvenuti-Ferreira \& Coelho 2009).

Phytosociological parameters of frequency, density and cover, the importance value (IV), Shannon index of diversity (H') and Pielou's evenness index ( $\mathrm{J}$ ') were determined according to Brower et al. (1998). The diversity was also quantified with $\mathrm{D}$, the effective number of species (Jost 2006), where $D=e^{H}$.

The richness of both areas was compared through the rarefaction curves, which were performed with EstimateS 8.00 (Colwell 2006). The number of randomizations was 50 without replacement, using bias-corrected formula CHAO 1 \& CHAO 2 (Chao 1987) except to the understory of the secondary forest area, considering the incidence distribution $\mathrm{CV} \%$ more than 0.5 for this case. The rarefaction curve of the tree strata was based on Benvenuti-Ferreira \& Coelho (2009) data, obtained through similar phytosociological method.

The percentage of similarity among communities and strata was calculated according to $P S=1-\Sigma\left|p_{i}-q_{i}\right| / 2$, where $p_{i}$ and $q_{i}$ are the proportion of the species $i$ on each community (Brower et al. 1998).

Species were identified with the assistance of experts, herbaria and literature (Sobral et al. 2006). The classification followed the APG II System (Souza \& Lorenzi 2008) and the nomenclature was according the International Plant Names Index (International... 2006). Voucher material was deposited in the Rogério Bueno Herbarium of the UNIJUÍ University (HUI). Psychotria fractistipula Smith, Klein \& Delprete and P. myriantha Cham. \& Schltdl. could not be differentiated during the phytosociological survey, in such way they are considered as just one species for calculation purposes.

The species classification into successional guilds followed Carvalho (1994) who had oriented his classification based on
Budowski (1965), also according with Ruchel et al. (2007) and authors observations. Pioneer, early secondary, late secondary and shade tolerant were the applied categories, the last one assumed here as equivalent to climax.

To designate the dispersion mode, the classification proposed by van Der Pijl (1982) was adopted, dividing the diaspore types according to the features of the dispersion unit. The zoochory was characterized by presence of potential rewards, such as arile, pigmentation and flesh pulp. The anemochory was identified by structures that favor the wind dispersion such as wings. The autochory was characterized by the presence of autogenous adaptations to dispersion or, oppositely, absence of adaptations to exogenous vectors. The classification of species was obtained by direct observation and with assistance of literature (Oliveira \& Moreira 1992, Barroso et al. 1999, Morellato \& Leitão-Filho 1992, Ruchel et al. 2007).

The leguminous tree species were classified as mutualist when the presence of bacterial $\mathrm{N}$ fixation was assigned by the literature (De Faria et al. 1989, Carvalho 1994).

\section{Results}

\section{Soils}

The soils in the Mato Silva are acid, with low levels of the macronutrients $\mathrm{P}, \mathrm{K}, \mathrm{Ca}$ and $\mathrm{Mg}$ and high levels of $\mathrm{Al}$. On the other hand, the early stages of succession presented higher levels of $\mathrm{pH}$, $\mathrm{CEC}$ and $\mathrm{V} \%$ than late successional forest (Table 1). $\mathrm{Cu}$ tends to decrease in the soil along the succession, with the lowest value in the late successional forest.

\section{Plant diversity in the understory}

The sampling consisted of 384 individuals in the late successional forest (LF) and 284 in the young secondary forest (SF). Both formations encompassed 80 different species in the understory (15 as shrub species), and only eight species were found in the two areas.

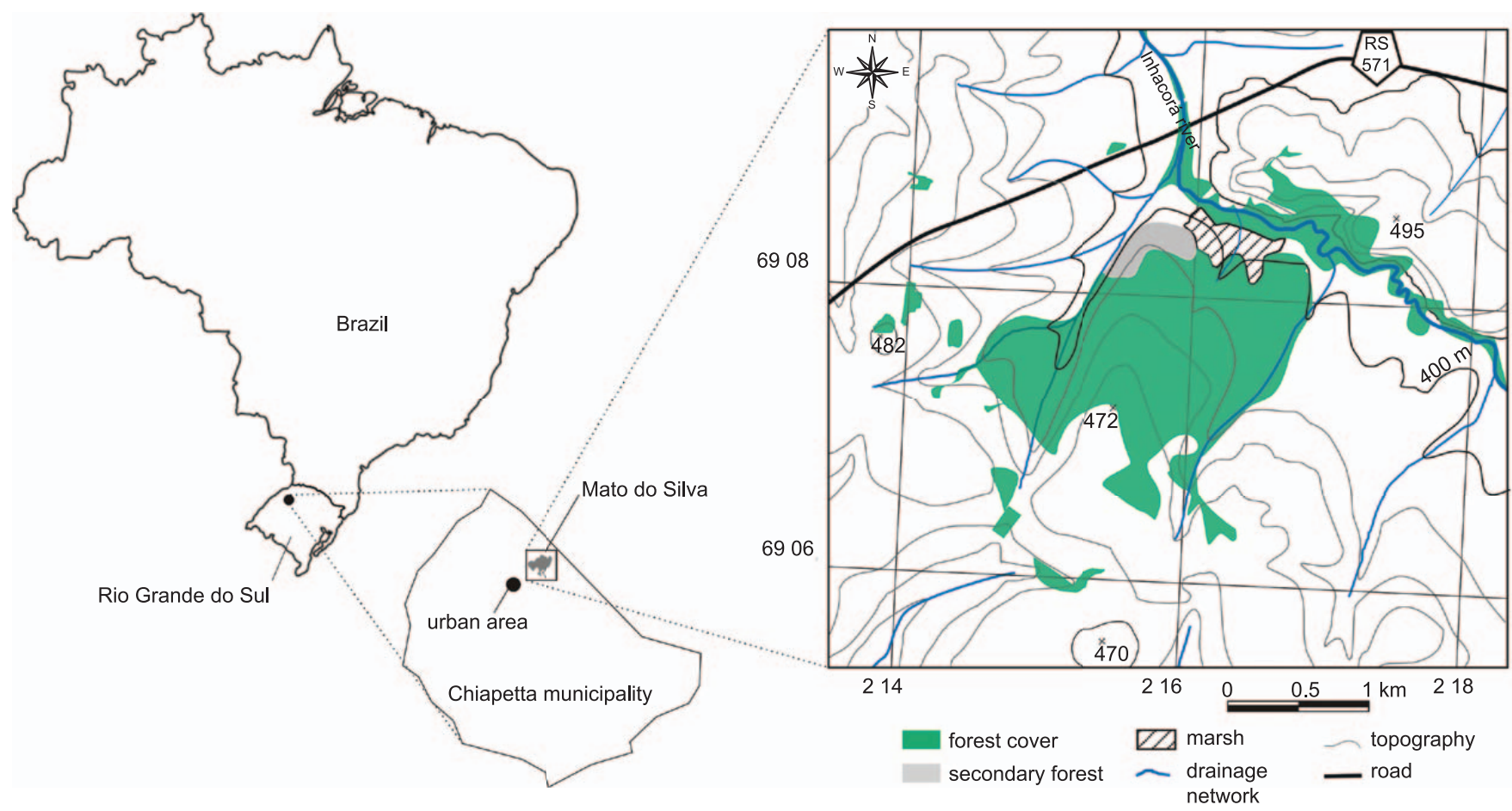

Figure 1. Mato do Silva forest remnant location in Chiapetta municipality, State of Rio Grande do Sul, Brazil. Based on map from DSG - Diretoria do Serviço Geográfico Brasileiro (1977). 
Coelho, G.C. et al.

Table 1. Physicochemical profile of the soil (median values to P and average values to all others), Mato do Silva forest remnant, Chiapetta, State of Rio Grande do Sul, Brazil.

\begin{tabular}{|c|c|c|c|c|c|}
\hline & & ES & SF & LF & \\
\hline $\mathrm{pH}$ & & $5.9 \pm 0.3^{\mathrm{a}}$ & $5.4 \pm 0.4^{\mathrm{ab}}$ & $4.9 \pm 0.3^{c}$ & $*$ \\
\hline $\mathrm{P}^{1}$ & & 4.2 & 3.4 & $<3.0$ & \\
\hline $\mathrm{K}$ & & $73.0 \pm 32.9$ & $53.7 \pm 19.3$ & $55.3 \pm 3.8$ & \\
\hline S & $\mathrm{mg} / \mathrm{dm}^{3}$ & $4.3 \pm 1.1$ & $4.2 \pm 2.9$ & $14.5 \pm 11.8$ & \\
\hline $\mathrm{Cu}$ & & $8.3 \pm 2.2^{\mathrm{a}}$ & $4.5 \pm 0.2^{\mathrm{ab}}$ & $3.2 \pm 0.7^{c}$ & $*$ \\
\hline $\mathrm{Zn}$ & & $4.9 \pm 2.8$ & $2.1 \pm 0.8$ & $2.7 \pm 1.0$ & \\
\hline $\mathrm{Mn}$ & & $54.1 \pm 39.5$ & $50.0 \pm 10.6$ & $66.2 \pm 24.5$ & \\
\hline $\mathrm{Ca}$ & & $7.0 \pm 0.8$ & $6.7 \pm 2.1$ & $5.1 \pm 2.7$ & \\
\hline $\mathrm{Mg}$ & $\mathrm{cmol}_{\mathrm{c}} / \mathrm{dm}^{3}$ & $2.9 \pm 0.3$ & $3.1 \pm 1.0$ & $2.0 \pm 1.0$ & \\
\hline $\mathrm{Al}$ & & $0.0 \pm 0.0^{\mathrm{a}}$ & $0.1 \pm 0.2^{\mathrm{ab}}$ & $0.7 \pm 0.5^{\mathrm{c}}$ & $*$ \\
\hline $\mathrm{H}+\mathrm{Al}$ & & $4.2 \pm 0.3$ & $6.2 \pm 3.1$ & $10.6 \pm 5.8$ & \\
\hline $\mathrm{CEC}(\mathrm{pH}=7.0)$ & & $14.6 \pm 1.3$ & $16.1 \pm 1.7$ & $17.5 \pm 5.6$ & \\
\hline V\% & & $71.0 \pm 3.2^{\mathrm{a}}$ & $62.0 \pm 8.8^{\mathrm{ab}}$ & $41.0 \pm 14.1^{\mathrm{c}}$ & $*$ \\
\hline $\mathrm{M} \%{ }^{2}$ & & $0.0 \pm 0.0^{\mathrm{a}}$ & $1.8 \pm 3.1^{\mathrm{ab}}$ & $10.8 \pm 8.0^{c}$ & $*$ \\
\hline O.M. (\%) & & $4.0 \pm 0.9$ & $4.3 \pm 0.9$ & $4.2 \pm 0.7$ & \\
\hline
\end{tabular}

$\mathrm{ES}=$ early secondary phase with grasses and shrubs, $\mathrm{SF}=$ young secondary forest and LF $=$ late successional forest. $*$ Means $p<0.05$ (in the one-way ANOVA), different letters indicate Tukey test differences $(p<0.05)$. ${ }^{1}$ Median values. ${ }^{2} \mathrm{Al}$ saturation

The 68 species of the LF belonged to 49 genera and 28 families (Table 2). The families with highest specific richness were Fabaceae (nine species), Solanaceae (eight), Lauraceae, Meliaceae and Sapindaceae (five species each).

The 20 species recorded in the SF belonged to 19 genera and 14 families (Table 2). The richest families were Fabaceae, Solanaceae (three species each one), Myrtaceae and Rutaceae (two species each one).

Only one shrub species was observed in SF and 14 of them were observed in LF. The outstanding shrub genera were Psychotria and Solanum with three species each one (Tables 2, 3 and 4).

The density of LF was 5,121 individuals per hectare, while SF had 838 individuals per hectare. The Shannon index (H') was 3.45 in the LF and 2.42 in the SF, corresponding to 35 and 11 effective number of species (D), respectively. The evenness index $\mathrm{J}$ was 0.85 to $\mathrm{LF}$ and 0.80 to SF.

The rarefaction curves for the understory indicate a higher expectation of richness to LF than to SF. In addition, the expected richness of the understory of LF was higher than of the tree strata in the same area. However, when the shrub species were excluded, LF understory presented a lower diversity than the tree strata (Figure 2).

In LF understory, Psychotria leiocarpa, Sorocea bonplandii, Cupania vernalis and Nectandra megapotamica had the highest importance values (Table 3). The first two are shade tolerant species, and the other two are secondary species. In the SF the species with the highest importance values were Ateleia glazioveana, Helietta apiculata and Schinus terebinthifolius (Table 4), all pioneer tree species. The species with highest density in the SF were A. glazioveana, Solanum compressum L. B. Sm. \& Downs and $S$. mauritianum, representing more than $50 \%$ of the individuals (Table 4).

Certain dominant species in the SF understory such as A. glazioveana, S. terebinthifolius, Zanthoxylum rhoifolium Lam. and S. compressum (Table 4) were absent in the LF understory (Table 2).

The SF understory presented higher similarity with the tree strata of the LF $(P S=15.0)$ than with the LF understory $(P S=8.2)$. On the other hand, the LF understory showed the highest similarity with the LF tree strata $(P S=39.9)$.

\section{Dispersion mechanisms, guilds and biological nitrogen fixation}

Zoochory was the dominant dispersion mode considering the relative abundance and richness in the understory of both successional stages. Zoochorous species also presented highest basal area in LF while wind-dispersed species were dominant in SF (Table 5).

Shade tolerant species predominates in LF with $40 \%$ of the individuals, while pioneer species were the major guild in SF understory, with $49 \%$ (Figure 3).

The nitrogen-fixing leguminous trees (NFL) were Albizia edwallii (Hoehne) J. W. Grimes, Parapiptadenia rigida (Benth.) Brenan (Mimosoidae), A. glazioveana, Lonchocarpus campestris Mart. ex Benth., Machaerium paraguariense Hassl., M. stipitatum Vogel and Myrocarpus frondosus Freire Allemão (Faboideae). The proportion of NFL was higher in SF in terms of basal area and relative density (Table 6) than in LF. Nevertheless, in SF this group was composed only by two species, and more than $95 \%$ of the individuals were A. glazioveana (Table 4). While the relative density of NFL tree species decreased in the late successional forest, the relative dominance was higher in the late successional forest understory than in the late successional tree strata (Table 6).

\section{Discussion}

Soil fertility of Mato do Silva could be considered medium when compared with other Semideciduous Forest sites in Rio Grande do Sul, for instance in Camaquã that had lower values of bases (except to potassium) and CEC (Jarenkow \& Jurinitz 2003). On the other hand, the Forest from Vale do Sol presented higher values to almost all nutrients (Jarenkow \& Waechter 2001) when compared to Mato do Silva LF.

The soil in the LF presented the lowest fertility and $\mathrm{pH}$. These results agree with those obtained by Bautista-Cruz \& del Castillo (2005) in secondary succession of a Mexican Tropical Montane Cloud Forest grown in Inceptisoils. However, the study by Aweto (1981) with secondary succession in highly weathered tropical soils from Nigeria indicates an increase on nutrients $(\mathrm{K}, \mathrm{Mg}, \mathrm{N}, \mathrm{P})$ and $\mathrm{CEC}$ in the topsoil $(0-10 \mathrm{~cm})$ during the initial decade (but no significant changes in depths below $10 \mathrm{~cm}$ ). These results could be explained by 
Understory structure in Semi-deciduous Seasonal Forest

Table 2. Shrub and tree species in the understory of the late successional forest (LF) and in the secondary forest (SF), Mato do Silva, Chiapetta, State of Rio Grande do Sul, Brazil.

\begin{tabular}{|c|c|c|c|}
\hline Families & Species & LF & SF \\
\hline \multirow[t]{2}{*}{ Acanthaceae } & Justicia brasiliana Roth & $\mathrm{X}$ & - \\
\hline & Ruellia brevifolia (Pohl) C. Ezcurra & $\mathrm{X}$ & - \\
\hline Anacardiaceae & Schinus terebinthifolius Raddi & - & $\mathrm{X}$ \\
\hline Annonaceae & Rollinia rugulosa Schltdl. & $\mathrm{X}$ & - \\
\hline \multirow[t]{2}{*}{ Aquifoliaceae } & Ilex brevicuspis Reissek & $\mathrm{X}$ & - \\
\hline & Ilex paraguariensis A.St.-Hil. & $\mathrm{X}$ & $\mathrm{X}$ \\
\hline Asteraceae & Senecio brasiliensis (Spreng. ex Baker) Less. & $\mathrm{X}$ & - \\
\hline Boraginaceae & Cordia americana (L.) Gottschling \& J.E.Mill. & $\mathrm{X}$ & $\mathrm{X}$ \\
\hline Cannabaceae & Celtis iguanaea (Jacq.) Sarg. & $\mathrm{X}$ & - \\
\hline \multirow[t]{2}{*}{ Celastraceae } & Maytenus dasyclados Mart. & $\mathrm{X}$ & - \\
\hline & Schaefferia argentinensis Speg. & $\mathrm{X}$ & - \\
\hline Erythroxylaceae & Erythroxylum deciduum A. St.-Hilaire & - & $\mathrm{X}$ \\
\hline \multirow[t]{4}{*}{ Euphorbiaceae } & Acalypha gracilis Spreng. & $\mathrm{X}$ & - \\
\hline & Bernardia pulchella (Baill.) Müll. Arg. & $\mathrm{X}$ & - \\
\hline & Gymnanthes concolor Spreng. & $\mathrm{X}$ & - \\
\hline & Sebastiania commersoniana (Baill.) L.B.Sm. \& Downs & - & $\mathrm{X}$ \\
\hline \multirow[t]{9}{*}{ Fabaceae } & Albizia edwallii (Hoehne) J.W.Grimes & $\mathrm{X}$ & - \\
\hline & Apuleia leiocarpa (Vogel) J. F. Macbride & $\mathrm{X}$ & - \\
\hline & Ateleia glazioveana Baill. & - & $\mathrm{X}$ \\
\hline & Gledtisia amorphoides (Griseb.) Taubert & - & $\mathrm{X}$ \\
\hline & Lonchocarpus campestris Mart. ex Benth. & $\mathrm{X}$ & - \\
\hline & Machaerium paraguariense Hassl. & $\mathrm{X}$ & - \\
\hline & Machaerium stipitatum Vogel & $\mathrm{X}$ & $\mathrm{X}$ \\
\hline & Myrocarpus frondosus Allem. & $\mathrm{X}$ & - \\
\hline & Parapiptadenia rigida (Benth.) Brenan & $\mathrm{X}$ & - \\
\hline Lamiaceae & Vitex megapotamica (Spreng.) Moldenke & $\mathrm{X}$ & - \\
\hline \multirow[t]{5}{*}{ Lauraceae } & Endlicheria paniculata (Spreng.) J.F.Macbr. & $\mathrm{X}$ & - \\
\hline & Nectandra lanceolata Nees & $\mathrm{X}$ & - \\
\hline & Nectandra megapotamica (Spreng.) Mez & $\mathrm{X}$ & - \\
\hline & Ocotea diospyrifolia (Meisn.) Mez & $\mathrm{X}$ & - \\
\hline & Ocotea puberula (Rich.) Nees & $\mathrm{X}$ & $\mathrm{X}$ \\
\hline Malvaceae & Triumfetta semitriloba Jacq. & $\mathrm{X}$ & - \\
\hline \multirow[t]{5}{*}{ Meliaceae } & Cabralea canjerana (Vell.) Mart. & $X$ & - \\
\hline & Cedrela fissilis Vell. & $X$ & - \\
\hline & Trichilia catigua A. Juss. & $\mathrm{X}$ & - \\
\hline & Trichilia claussenii C. DC. & $\mathrm{X}$ & - \\
\hline & Trichilia elegans A. Juss. & $\mathrm{X}$ & - \\
\hline Moraceae & Sorocea bonplandii (Baill.) Burger, Lanjouw \& Boer & $\mathrm{X}$ & - \\
\hline \multirow[t]{2}{*}{ Myrsinaceae } & Myrsine coriacea (Sw.) R.Br. ex Roem. \& Schult. & $\mathrm{X}$ & - \\
\hline & Myrsine laetevirens (Mrz) Arechav. & $X$ & - \\
\hline \multirow[t]{7}{*}{ Myrtaceae } & Calyptranthes concinna DC. & $\mathrm{X}$ & $\mathrm{X}$ \\
\hline & Campomanesia guazumifolia (Cambess.) O. Berg & $\mathrm{X}$ & - \\
\hline & Campomanesia xanthocarpa O. Berg & $\mathrm{X}$ & - \\
\hline & Eugenia involucrata DC. & $\mathrm{X}$ & - \\
\hline & Eugenia rostrifolia D. Legrand & $\mathrm{X}$ & - \\
\hline & Eugenia uniflora $\mathrm{L}$. & $\mathrm{X}$ & - \\
\hline & Myrcia bombicyna (O.Berg.) Kiaersk. & - & $\mathrm{X}$ \\
\hline Piperaceae & Piper aduncum L. & $\mathrm{X}$ & - \\
\hline Polygonaceae & Ruprechtia laxiflora Meisn. & - & $\mathrm{X}$ \\
\hline Rosaceae & Prunus myrtifolia (L.) Urb. & $\mathrm{X}$ & $\mathrm{X}$ \\
\hline \multirow[t]{2}{*}{ Rubiaceae } & Psychotria fractistipula Smith, Klein \& Delprete & $\mathrm{X}$ & - \\
\hline & Psychotria leiocarpa Cham. \& Schltdl. & $\mathrm{X}$ & - \\
\hline
\end{tabular}


Coelho, G.C. et al.

Table 2. Continued...

\begin{tabular}{|c|c|c|c|}
\hline Families & Species & LF & SF \\
\hline & Psychotria myriantha Cham. \& Schltdl. & $\mathrm{X}$ & - \\
\hline & Rudgea parquioides (Cham.) Müll. Arg. & $\mathrm{X}$ & - \\
\hline \multirow[t]{4}{*}{ Rutaceae } & Balfourodendron riedelianum (Engl.) Engl. & $\mathrm{X}$ & - \\
\hline & Helietta apiculata Benth. & $\mathrm{X}$ & $\mathrm{X}$ \\
\hline & Pilocarpus pennatifolius Lam. & $\mathrm{X}$ & - \\
\hline & Zanthoxylum rhoifolium Lam. & - & $\mathrm{X}$ \\
\hline \multirow[t]{3}{*}{ Salicaceae } & Banara tomentosa Clos & $\mathrm{X}$ & - \\
\hline & Casearia decandra Jacq. & $\mathrm{X}$ & - \\
\hline & Casearia sylvestris $\mathrm{Sw}$. & - & $\mathrm{X}$ \\
\hline \multirow[t]{5}{*}{ Sapindaceae } & Allophylus edulis (A. St.-Hil., Camb. \& Juss.) Radlk. & $\mathrm{X}$ & - \\
\hline & Allophylus guaraniticus (A. St.-Hil.) Radlk. & $\mathrm{X}$ & - \\
\hline & Cupania vernalis Camb. & $\mathrm{X}$ & - \\
\hline & Diatenopteryx sorbifolia Radlk. & $\mathrm{X}$ & - \\
\hline & Matayba elaeagnoides Radlk. & $\mathrm{X}$ & - \\
\hline \multirow[t]{2}{*}{ Sapotaceae } & Chrysophyllum gonocarpum (Mart. \& Eichler) Engl. & $\mathrm{X}$ & - \\
\hline & Chrysophyllum marginatum (Hook. \& Arn.) Radlk. & $\mathrm{X}$ & - \\
\hline \multirow[t]{2}{*}{ Simaroubaceae } & Castela tweediei Planch. & - & $\mathrm{X}$ \\
\hline & Picrasma crenata (Vell.) Engl. & $\mathrm{X}$ & - \\
\hline \multirow[t]{8}{*}{ Solanaceae } & Capsicum flexuosum Sendtn. & $\mathrm{X}$ & - \\
\hline & Cestrum intermedium Sendtn. & - & $\mathrm{X}$ \\
\hline & Solanum atropurpureum Schrank & $\mathrm{X}$ & - \\
\hline & Solanum compressum L. B. Sm. \& Downs & - & $\mathrm{X}$ \\
\hline & Solanum gemellum Mart. ex Sendt. & $\mathrm{X}$ & - \\
\hline & Solanum mauritianum Scop. & $\mathrm{X}$ & $\mathrm{X}$ \\
\hline & Solanum nigrescens M. Martens \& Galeotti & $\mathrm{X}$ & - \\
\hline & Solanum pseudo-quina A. St. -Hil. & $\mathrm{X}$ & - \\
\hline Styracaceae & Styrax leprosus Hook. \& Arn. & $\mathrm{X}$ & - \\
\hline Symplocaceae & Symplocos cf. tetrandra Mart. ex Miq. & $\mathrm{X}$ & - \\
\hline
\end{tabular}

Table 3. Phytossociological data (\%), successional guild (G) and dispersion mode (D) of shrub and tree species in the understory of the late successional forest, Chiapetta, State of Rio Grande do Sul, Brazil (Shrub species in bold).

\begin{tabular}{|c|c|c|c|c|c|c|}
\hline Species & $\mathbf{D R}^{1}$ & $\mathbf{F R}^{2}$ & $\mathbf{C R}^{3}$ & $\mathrm{VI}^{4}$ & $\mathbf{G}^{5}$ & $\mathrm{D}^{6}$ \\
\hline Psychotria leiocarpa & 10.94 & 8.68 & 2.83 & 7.48 & $\mathrm{E}$ & $\mathrm{ZO}$ \\
\hline Cupania vernalis & 10.68 & 10.29 & 10.06 & 10.34 & SI & $\mathrm{ZO}$ \\
\hline Nectandra megapotamica & 5.99 & 6.75 & 4.65 & 5.80 & SL & $\mathrm{ZO}$ \\
\hline Sorocea bonplandii & 5.99 & 4.50 & 6.94 & 5.81 & $\mathrm{E}$ & $\mathrm{ZO}$ \\
\hline Trichilia elegans & 5.73 & 5.79 & 7.82 & 6.44 & $\mathrm{E}$ & $\mathrm{ZO}$ \\
\hline Matayba elaeagnoides & 4.69 & 4.82 & 4.15 & 4.55 & SI & $\mathrm{AN}$ \\
\hline Solanum mauritianum & 4.17 & 3.54 & 2.69 & 3.46 & $\mathrm{P}$ & $\mathrm{ZO}$ \\
\hline Balfourodendron riedelianum & 3.39 & 3.22 & 2.11 & 2.90 & SL & AN \\
\hline Diatenopteryx sorbifolia & 3.39 & 3.54 & 7.24 & 4.72 & SI & AN \\
\hline Bernardia pulchella & 2.86 & 2.89 & 3.60 & 3.12 & $\mathrm{E}$ & $\mathrm{AU}$ \\
\hline Gymnanthes concolor & 2.60 & 1.93 & 2.26 & 2.26 & $\mathrm{E}$ & $\mathrm{AU}$ \\
\hline Piper aduncum & 2.60 & 2.57 & 2.05 & 2.41 & $\mathrm{E}$ & $\mathrm{ZO}$ \\
\hline Psychotria spp. ${ }^{\Delta}$ & 2.60 & 2.57 & 0.64 & 1.94 & $\mathrm{E}$ & $\mathrm{ZO}$ \\
\hline Prunus myrtifolia & 1.82 & 1.93 & 0.26 & 1.34 & SI & $\mathrm{ZO}$ \\
\hline Eugenia rostrifolia & 1.56 & 1.61 & 2.29 & 1.82 & SL & $\mathrm{ZO}$ \\
\hline Justicia brasiliana & 1.56 & 0.96 & 0.34 & 0.96 & $\mathrm{E}$ & $\mathrm{ZO}$ \\
\hline
\end{tabular}

${ }^{1}$ DR: relative density; ${ }^{2} \mathrm{FR}$ : relative frequency; ${ }^{3} \mathrm{CR}$ : relative coverage $;{ }^{4} \mathrm{VI}$ : importance percentage; ${ }^{5}$ successional guild: $\mathrm{P}=$ pioneer, $\mathrm{SI}=$ early secondary, $\mathrm{SL}=$ late secondary, $\mathrm{E}=$ shade tolerant; ${ }^{6}$ dispersion: $\mathrm{AN}=$ anemochory, $\mathrm{AU}=$ autochory, $\mathrm{ZO}=$ zoochory. ${ }^{\Delta}$ Psychotria fractistipula $+P$. myriantha (see Methods). 
Table 3. Continued...

\begin{tabular}{|c|c|c|c|c|c|c|}
\hline Species & $\mathbf{D R}^{1}$ & $\mathbf{F R}^{2}$ & $\mathbf{C R}^{3}$ & $\mathrm{VI}^{4}$ & $\mathbf{G}^{5}$ & $\mathbf{D}^{6}$ \\
\hline Apuleia leiocarpa & 1.30 & 1.29 & 0.23 & 0.94 & SL & AN \\
\hline Cabralea canjerana & 1.30 & 1.28 & 2.95 & 5.53 & SL & $\mathrm{ZO}$ \\
\hline Machaerium stipitatum & 1.30 & 1.61 & 1.23 & 1.38 & SI & AN \\
\hline Myrocarpus frondosus & 1.30 & 0.96 & 0.94 & 1.07 & SL & $\mathrm{ZO}$ \\
\hline Solanum gemellum & 1.30 & 1.29 & 0.44 & 1.01 & SL & $\mathrm{ZO}$ \\
\hline Solanum pseudo-quina & 1.30 & 1.61 & 2.75 & 1.89 & SI & $\mathrm{ZO}$ \\
\hline Campomanesia guazumifolia & 1.04 & 1.29 & 0.55 & 0.96 & SL & $\mathrm{ZO}$ \\
\hline Chrysophyllum gonocarpum & 1.04 & 1.29 & 0.94 & 1.09 & $\mathrm{E}$ & $\mathrm{ZO}$ \\
\hline Myrsine coriacea & 1.04 & 1.29 & 0.23 & 0.85 & $\mathrm{P}$ & AN \\
\hline Ocotea diospyrifolia & 1.04 & 1.29 & 2.78 & 1.70 & SL & $\mathrm{ZO}$ \\
\hline Ocotea puberula & 1.04 & 1.29 & 0.28 & 0.87 & SI & $\mathrm{ZO}$ \\
\hline Rollinia rugulosa & 1.04 & 0.96 & 0.40 & 0.80 & SI & $\mathrm{ZO}$ \\
\hline Campomanesia xanthocarpa & 0.78 & 0.96 & 2.89 & 1.55 & SI & $\mathrm{ZO}$ \\
\hline Ilex brevicuspis & 0.78 & 0.96 & 1.31 & 1.02 & SI & $\mathrm{AN}$ \\
\hline Machaerium paraguariense & 0.78 & 0.96 & 3.17 & 1.64 & SI & AN \\
\hline Myrsine laetevirens & 0.78 & 0.96 & 0.17 & 0.64 & SI & $\mathrm{ZO}$ \\
\hline Parapiptadenia rigida & 0.78 & 0.96 & 3.26 & 1.67 & SI & AN \\
\hline Solanum atropurpureum & 0.78 & 0.96 & 0.28 & 0.67 & SI & $\mathrm{ZO}$ \\
\hline Symplocos cf. tetrandra & 0.78 & 0.96 & 0.26 & 0.67 & SI & $\mathrm{ZO}$ \\
\hline Albizia edwallii & 0.52 & 0.64 & 4.07 & 1.75 & SI & $\mathrm{AN}$ \\
\hline Banara tomentosa & 0.52 & 0.64 & 0.27 & 0.48 & SL & $\mathrm{ZO}$ \\
\hline Calyptranthes concinna & 0.52 & 0.64 & 0.21 & 0.46 & SL & $\mathrm{ZO}$ \\
\hline Capsicum flexuosum & 0.52 & 0.64 & 0.24 & 0.47 & $\mathrm{E}$ & $\mathrm{ZO}$ \\
\hline Celtis iguanae & 0.52 & 0.64 & 0.08 & 0.42 & SI & $\mathrm{ZO}$ \\
\hline Eugenia uniflora & 0.52 & 0.64 & 0.17 & 0.44 & SI & $\mathrm{ZO}$ \\
\hline Nectandra lanceolata & 0.52 & 0.64 & 0.10 & 0.42 & SI & $\mathrm{ZO}$ \\
\hline Rudgea parquioides & 0.52 & 0.64 & 0.49 & 0.55 & SL & $\mathrm{ZO}$ \\
\hline Ruellia sanguinea & 0.52 & 0.32 & 0.10 & 0.31 & $\mathrm{E}$ & $\mathrm{AU}$ \\
\hline Schaefferia argentinensis & 0.52 & 0.64 & 0.21 & 0.46 & $\mathrm{E}$ & $\mathrm{ZO}$ \\
\hline Senecio brasiliensis & 0.52 & 0.64 & 0.27 & 0.48 & $\mathrm{P}$ & AN \\
\hline Acalypha gracilis & 0.26 & 0.32 & 0.13 & 0.24 & $\mathrm{E}$ & $\mathrm{AU}$ \\
\hline Alophyllus edulis & 0.26 & 0.32 & 0.05 & 0.21 & SL & $\mathrm{ZO}$ \\
\hline Alophyllus guaraniticus & 0.26 & 0.32 & 0.06 & 0.21 & $\mathrm{E}$ & $\mathrm{ZO}$ \\
\hline Casearia decandra & 0.26 & 0.32 & 0.16 & 0.25 & SL & $\mathrm{ZO}$ \\
\hline Cedrela fissilis & 0.26 & 0.32 & 0.04 & 0.21 & SI & AN \\
\hline Chrysophyllum marginatum & 0.26 & 0.32 & 2.51 & 1.03 & SI & $\mathrm{ZO}$ \\
\hline Cordia americana & 0.26 & 0.32 & 1.58 & 0.72 & SI & AN \\
\hline Endlicheria paniculata & 0.26 & 0.32 & 0.04 & 0.21 & SL & $\mathrm{ZO}$ \\
\hline Eugenia involucrata & 0.26 & 0.32 & 0.53 & 0.37 & SL & $\mathrm{ZO}$ \\
\hline Helietta apiculata & 0.26 & 0.32 & 0.06 & 0.22 & $\mathrm{P}$ & $\mathrm{AU}$ \\
\hline Ilex paraguariensis & 0.26 & 0.32 & 0.87 & 0.48 & $\mathrm{E}$ & $\mathrm{ZO}$ \\
\hline Lonchocarpus campestris & 0.26 & 0.32 & 2.64 & 1.07 & SI & $\mathrm{AU}$ \\
\hline Maytenus dasyclados & 0.26 & 0.32 & 0.24 & 0.27 & $\mathrm{E}$ & $\mathrm{ZO}$ \\
\hline Picrasma crenata & 0.26 & 0.32 & 0.12 & 0.23 & SL & $\mathrm{ZO}$ \\
\hline Pilocarpus pennatifolius & 0.26 & 0.32 & 1.03 & 0.54 & $\mathrm{E}$ & $\mathrm{AU}$ \\
\hline Solanum nigrescens & 0.26 & 0.32 & 0.08 & 0.22 & $\mathrm{E}$ & $\mathrm{ZO}$ \\
\hline Styrax leprosum & 0.26 & 0.32 & 0.92 & 0.50 & SL & $\mathrm{ZO}$ \\
\hline Trichilia catigua & 0.26 & 0.32 & 0.11 & 0.23 & $\mathrm{E}$ & $\mathrm{ZO}$ \\
\hline Trichilia claussenii & 0.26 & 0.32 & 0.48 & 0.35 & SL & $\mathrm{ZO}$ \\
\hline Triumphetta semitriloba & 0.26 & 0.32 & 0.03 & 0.20 & $\mathrm{E}$ & $\mathrm{ZO}$ \\
\hline Vitex megapotamica & 0.26 & 0.32 & 0.06 & 0.21 & SI & $\mathrm{ZO}$ \\
\hline
\end{tabular}

${ }^{1}$ DR: relative density; ${ }^{2} \mathrm{FR}$ : relative frequency; ${ }^{3} \mathrm{CR}$ : relative coverage $;{ }^{4} \mathrm{VI}$ : importance percentage $;{ }^{5}$ successional guild: $\mathrm{P}=$ pioneer, $\mathrm{SI}=$ early secondary, $\mathrm{SL}=$ late secondary, $\mathrm{E}=$ shade tolerant; ${ }^{6}$ dispersion: $\mathrm{AN}=$ anemochory, $\mathrm{AU}=$ autochory, $\mathrm{ZO}=$ zoochory. ${ }^{\Delta}$ Psychotria fractistipula $+P$. myriantha (see Methods). 
the transference of nutrients to the topsoil via litter deposition. The increment (or decrease) of nutrient availability in the topsoil could be related to the balance between aboveground growth and biomass accumulation, in one side, and litter production and decomposition rates, in the other (Aweto 1981). In other words, if the transference of nutrients from topsoil to the aboveground biomass predominates in relation to the nutrients uptake from the deep soil and their transference to the topsoil via litter deposition, a decrease in topsoil fertility during succession could be observed.

Fabaceae was the distinguished family in both sampled forests. Such dominance was also observed on LF tree strata of Mato do Silva (Benvenuti-Ferreira \& Coelho 2009). The predominance of Fabaceae is typical in the Seasonal Forest from Southern Brazil

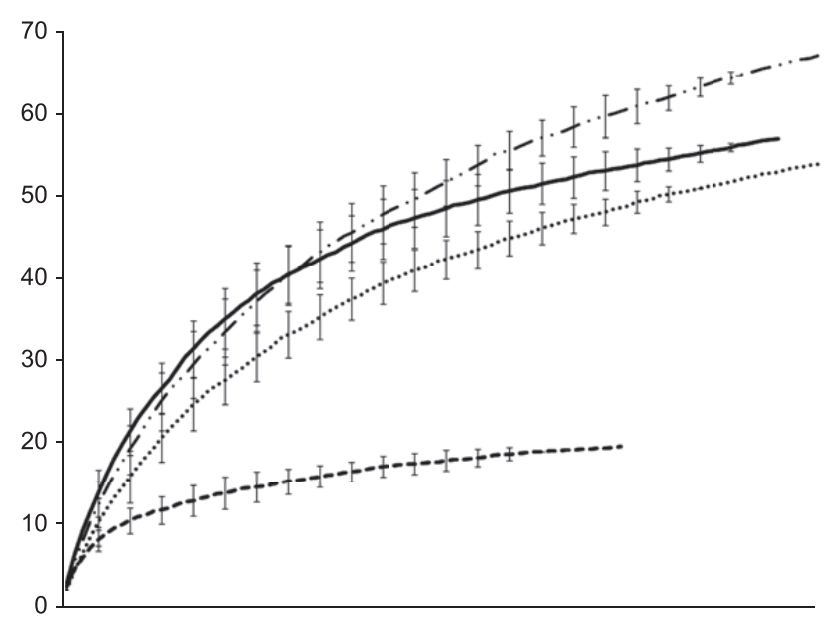

Figure 2. Rarefaction curves of understory of the late successional forest (dotted/dashed line), tree strata of the late successional forest $(\mathrm{DBH} \geq 5 \mathrm{~cm}$; solid line), understory strata of the late successional forest without the shrub species (dotted line), and the understory of the secondary forest (dashed line).
(Vasconcellos et al. 1992, Bencke \& Soares 1998, Jarenkow \& Waechter 2001, Giehl et al. 2007). High richness of Fabaceae is related to the immigration corridor of tropical tree species delineated by the Seasonal Forest of the upper Uruguay River Basin, which permits the arrival of species from North towards South (Rambo 1951, Jarenkow \& Jurinitz 2003). The dominance of Fabaceae in Seasonal Forest is supposedly due to the low levels of $\mathrm{N}$ in that biome (Jarenkow \& Waechter 2001), but such association was not investigated in detail.

The relative density of nitrogen-fixing leguminous tree was lower in the late successional forest. Nevertheless, the difference between successional phases concerning the presence of Fabaceae with biological nitrogen fixation remains unclear, since there are only two species in the secondary forest and one of them, A. glazioveana, is decisively dominant. In Central Amazonia, the fraction of legume trees with biological nitrogen fixation is higher in the early phases of secondary forest than in the late successional forest (Gehring et al.

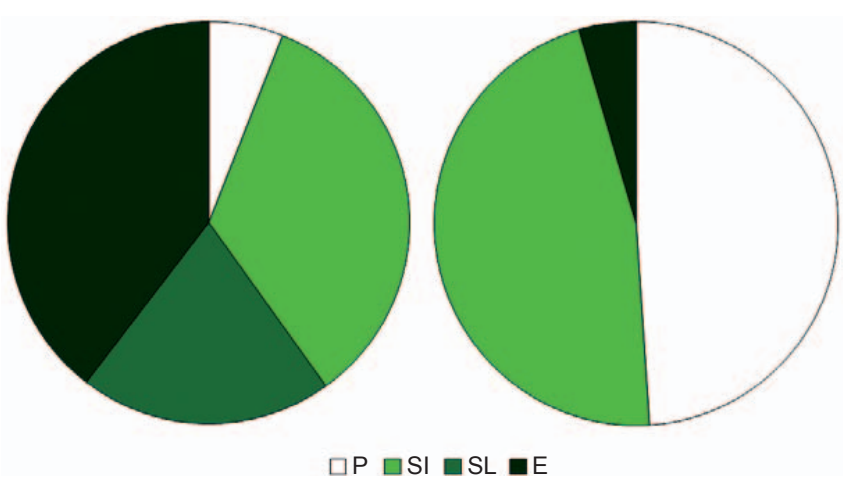

Figure 3. Proportion of pioneer species (P), early secondary species (SI), late secondary species (SL) and shade tolerant species (E) in the understory of the late successional forest (left) and in the understory of the secondary forest (right).

Table 4. Phytossociological data (\%), successional guild (G) and dispersion way (D) of shrub and tree species in the understory of the secondary forest, Chiapetta, State of Rio Grande do Sul, Brazil. The shrubby species are in bold.

\begin{tabular}{|c|c|c|c|c|c|c|}
\hline Species & $\mathbf{D R}^{1}$ & $\mathbf{F R}^{2}$ & $\mathbf{C R}^{3}$ & VI\% ${ }^{4}$ & $G^{5}$ & $\mathbf{D}^{6}$ \\
\hline Ateleia glazioveana & 18.31 & 17.54 & 16.62 & 17.49 & $\mathrm{P}$ & $\mathrm{AN}$ \\
\hline Solanum compressum & 15.14 & 12.32 & 1.07 & 9.51 & SI & $\mathrm{ZO}$ \\
\hline Solanum mauritianum & 12.32 & 12.32 & 4.34 & 9.66 & $\mathrm{P}$ & $\mathrm{ZO}$ \\
\hline Schinus terebinthifolius & 11.62 & 12.32 & 12.60 & 12.18 & $\mathrm{P}$ & $\mathrm{ZO}$ \\
\hline Zanthoxylum rhoifolium & 9.51 & 10.90 & 10.92 & 10.44 & SI & $\mathrm{ZO}$ \\
\hline Erythroxylum deciduum & 7.04 & 8.06 & 0.85 & 5.32 & SI & $\mathrm{ZO}$ \\
\hline Helietta apiculata & 6.69 & 6.64 & 31.35 & 14.89 & $\mathrm{P}$ & $\mathrm{AN}$ \\
\hline Ilex paraguariensis & 4.58 & 3.79 & 2.02 & 3.46 & $\mathrm{E}$ & $\mathrm{ZO}$ \\
\hline Cestrum intermedium & 4.23 & 3.79 & 0.16 & 2.73 & SI & $\mathrm{ZO}$ \\
\hline Prunus myrtifolia & 3.87 & 3.32 & 0.89 & 2.69 & SI & $\mathrm{ZO}$ \\
\hline Castela tweediei & 1.76 & 2.37 & 0.79 & 1.64 & SI & $\mathrm{ZO}$ \\
\hline Cordia americana & 1.06 & 1.42 & 0.86 & 1.11 & SI & $\mathrm{AN}$ \\
\hline Ocotea puberula & 0.70 & 0.95 & 0.14 & 0.60 & SI & $\mathrm{AN}$ \\
\hline Myrcia bombicina & 0.70 & 0.95 & 0.59 & 0.75 & SI & $\mathrm{ZO}$ \\
\hline Rupretchia laxiflora & 0.70 & 0.95 & 1.49 & 1.05 & SI & $\mathrm{ZO}$ \\
\hline Machaerium stipitatum & 0.35 & 0.47 & 0.06 & 0.30 & SI & $\mathrm{AU}$ \\
\hline Calypthranthes concinna & 0.35 & 0.47 & 0.88 & 0.57 & SI & $\mathrm{ZO}$ \\
\hline Gleditsia amorphoides & 0.35 & 0.47 & 2.15 & 0.99 & SI & $\mathrm{AU}$ \\
\hline Casearia sylvestris & 0.35 & 0.47 & 2.23 & 1.02 & SI & $\mathrm{ZO}$ \\
\hline Sebastiania commersoniana & 0.35 & 0.47 & 10.00 & 3.61 & SI & $\mathrm{AN}$ \\
\hline
\end{tabular}

${ }^{1} \mathrm{DR}$ : relative density; ${ }^{2} \mathrm{FR}$ : relative frequency; ${ }^{3} \mathrm{CR}$ : relative coverage; ${ }^{4} \mathrm{VI}$ : importance percentage; ${ }^{5}$ successional guild: $\mathrm{P}=$ pioneer, $\mathrm{SI}=$ early secondary, $\mathrm{SL}=$ late secondary, $\mathrm{E}=$ shade tolerant $;{ }^{6}$ dispersion: $\mathrm{AN}=$ anemochory; $\mathrm{AU}=$ autochory, $\mathrm{ZO}=$ zoochory. 
Table 5. Proportion of relative density (DR\%), richness (R\%) and relative coverage (CR\%) of the different dispersion modes in the understory of the late successional forest and in the secondary forest Mato do Silva, Chiapetta, 2008.

\begin{tabular}{lccc}
\hline \multicolumn{1}{c}{ Dispersion mechanisms } & DR(\%) & R\% & CR $(\%)$ \\
\hline Late sucessional forest & - & - & - \\
Anemochory & 19.0 & 19.7 & 28.9 \\
Autochory & 7.0 & 10.6 & 9.8 \\
Zoochory & 74.0 & 69.7 & 61.3 \\
Secondary forest & - & - & - \\
Anemochory & 27.1 & 25.0 & 59.0 \\
Autochory & 0.7 & 10.0 & 2.2 \\
Zoochory & 72.2 & 65.0 & 38.8 \\
\hline
\end{tabular}

Table 6. Proportion of Leguminous trees with biological fixation of nitrogen, $\mathrm{DR} \%=$ relative density, $\mathrm{R}=$ number of species and CR $\%=$ relative coverage Data of tree strata were obtained from Benvenuti-Ferreira \& Coelho (2009).

\begin{tabular}{lccc}
\hline & DR $(\boldsymbol{\%})$ & R & CR $(\boldsymbol{\%})$ \\
\hline Secondary forest understory & 18.7 & 2 & 17.2 \\
Late successional forest tree strata & 11.3 & 8 & 10.1 \\
Late successional forest understory & 5.0 & 6 & 15.3 \\
\hline
\end{tabular}

2005). Secondary succession in tropical forest recovers N-cycling processes. The $\mathrm{N}$ availability in the soil and the $\mathrm{N}$ concentration in the leaves increase with forest age (Davidson et al. 2007). Even when the total $\mathrm{N}$ of the ecosystem remains constant across a chronosequence, substantial transference of $\mathrm{N}$ occurs from the soil to the aboveground biomass and litter (Compton et al. 2007). In contrast, in calcareous and nutrient rich soils from Atlantic Forest, the forest succession can exhibit decrease in foliar nitrogen content along the succession process (Aidar et al. 2003). Notwithstanding, in that case the proportion of fixing leguminous trees was higher in the early ( 15 years) and intermediate ( 25 years) stands, decaying in the late succession phase (Aidar et al. 2001). As fixative bacteria implicates in cost to the vascular plants (Epstein \& Bloom 2005), which is a general rule in mutualism (Coelho 2009), the advantages of leguminous trees with biological fixation of $\mathrm{N}$ could be decreased along the succession, since the $\mathrm{N}$ availability is increased. Despite the nodulation presence, some leguminous species could uptake nitrogen from other chemical sources, reserving less energy to sustain the fixative bacteria.

A. glazioveana shows allelopathy in vitro, due to the presence of apolar compounds such as rutin and afrormosin, and polar compounds such as the non-protein amino acids (Marona et al. 2003). On the other hand, its fixation of atmospheric nitrogen through association with bacteria is very effective, allowing its use as green manure in different crops (Baggio \& Soares 2002, Baggio et al. 2002). In such way, A. glazioveana could promote facilitation rather than inhibition to other tree species along succession.

The richness of tree species in SF understory (19) is still low after 11 years of abandon, taking into account that the adjacent mature forest could provide a seed rain with high diversity. In a Bolivian dry forest growing in Inceptsols the secondary succession reaches $75 \%$ of the mature forest tree species richness after five years of abandon in a slash-and-burn agriculture context (Kennard 2002). Other studies in South America and Caribbean pointed that the secondary succession recovers at least $75 \%$ of species richness after a period from 5 to 60 years (Marín-Spiotta et al. 2007). There are not similar studies in the domain of Seasonal Subtropical forest, preventing a more appropriate comparison.

The past land use is decisive to the progress and features of secondary spontaneous succession (Uhl et al. 1988, Camargo et al. 2002). Some factors could have hypothetically contributed to the low floristic recovery in Mato do Silva, mainly the intensive previous agricultural activities, which altered physical soil structure and microflora. Otherwise, the soil analysis did not indicate mineral nutrient limitation in the early phase of succession. Other hypothesis could be evoked to explain the recruitment limitation, as competition with grasses and other herbs (Stevenson \& Smale 2005, Geeves et al. 2008) and insufficient seed rain (Clark et al. 1999, White et al. 2004).

There is a strong discrepancy between the understory of the two areas, with a predominance of pioneers and early secondary species in the secondary forest and the late successional species in the late successional forest. Some species such as Solanum compressum, Schinus terebinthifolius, Zanthoxylum rhoifolium and Castela tweediei could be connected to the early phase of secondary succession, disappearing when the forest reaches a later phase.

Only two of the 20 species with the highest importance values in the late successional forest understory ( $S$. mauritianum and Ilex paraguariensis A. St. Hil.) were sampled in the secondary forest. Meanwhile, among the 20 species with higher VI in the tree stratum of the late successional forest (Benvenuti-Ferreira \& Coelho 2009), seven species were recorded in the secondary forest understory, and 15 species occurred in the late successional forest understory. The understory of secondary forest presented high similarity with the tree strata of the late successional forest. Data indicate the LF tree strata maintain partially the species assemblage of early stages of succession. Among them, Gledtisia amorphoides could be emphasized, since it was included in the list of endangered species (Biodiversitas 2005), although the Brazilian government has indicated a lack of data pointing to the correct conservation status for this species (Brasil 2008). The regeneration of G. amorphoides could be restricted to early secondary stages or other open woodlands. In the future, if this successional condition disappears, the regeneration of this species will be prevented. Considering the low similarity in 
species composition between the secondary and late successional forest understories, the secondary forests could be important to the maintenance of landscape diversity, especially to some species which presented higher abundances in the early phases, and were virtually absent among the saplings in the late successional forest.

Rarefaction curves indicated a lower richness in the understory of the late sucessional forest when compared to the tree strata $(\mathrm{DBH} \geq 5.0 \mathrm{~cm})$, but only after shrub species exclusion. In addition, the diversity indexes showed a less diverse understory. In the LF tree strata, Benvenuti-Ferreira \& Coelho (2009) found H'= 3.61 $(\mathrm{D}=37)$ and $\mathrm{J}=0.89$, while the understory of late successional forest (without the exclusion of shrub species) had $\mathrm{H}^{\prime}=3.45(\mathrm{D}=31.5)$ and $\mathrm{J}=0.85$. Data could indicate a diversity decrease in the future of the late successional forest, as pointed out by Jarenkow \& Jurinitz (2003) to other Seasonal Forest site. On the other hand, the mortality of tree species could be density-dependent (Wills et al. 1997, 2006), suggesting different scenario, without diversity reduction in the tree strata of late successional forest, and it requires further analysis.

The zoochory is the dominant dispersion mode in both mature and secondary forest. This dominance is a strong tendency in tropical and subtropical forests (Howe \& Smallwood 1982, Morellato 1995, Budke et al. 2005), including the Seasonal Forests from Southern Brazil (Nascimento et al. 2000, Giehl et al. 2007, Ruchel et al. 2007). However, the wind-dispersed species presented the major fraction of the basal area (59\%) in the secondary forest. These trend of dominance are related especially with two species: $H$. apiculata and A. glazioveana, which reached $48 \%$ of basal area of the species assemblage. Anemochorous tree species tend to be tallest, since this dispersion strategy requires high wind availability (Giehl et al. 2007, Athayde et al. 2009). Tall trees allow less barriers and longer periods in the air to the diaspores, promoting farther dispersion (Horn et al. 2001). Anemochorous trees presented greater average of DBH than zoochorous trees in the Seasonal Forest of Mato do Silva (Benvenuti-Ferrreira \& Coelho 2009). Anemochorous tree species from the Seasonal Forest of Turvo State Park (Ruchel et al. 2007) were $26 \%$ of total richness but reached a sum of $42 \%$ of the total basal area, while the zoochoric species were $63 \%$ and summed $54 \%$ of the total basal area. In the tree strata of Mato do Silva late sucessional forest, anemochorous tree species reached $55 \%$ of the basal area with only $27 \%$ of richness. On the other hand, the zoochorous tree species encompassed $65 \%$ of richness and only $41 \%$ of the basal area (Benvenuti-Ferrreira \& Coelho 2009).

Forest restoration employing the attraction of dispersion agents such as birds can enhance the reintroduction of zoochorous tree species (Reis et al. 2003, Zanini \& Ganade 2005). However, it should be stressed the importance of anemochorous tree species to the Seasonal Forest structure (Vasconcellos et al. 1992, Athayde et al. 2009, Benvenuti-Ferrreira \& Coelho 2009) and regeneration in the secondary succession (according to present data). Alternative methods are required to reintroduce anemochorous tree species, when spontaneous recovery is hindered.

Restoration projects could be inspired in understory plant community of secondary forest instead of species from the late successional forest. Supposedly, the species of the secondary forest understory are better adapted to the initial ecological conditions of secondary succession. In addition, data indicates that the presence of Fabaceae with biological fixation of $\mathrm{N}$ is decisive in these early phases of forest regeneration. Especially, the ecological contribution of A. glazioveana deserves more attention.

As expected, the pioneers individuals predominate in the secondary forest while the shade tolerant is the most abundant guild in the late successional forest. I. paraguariensis is the single shade tolerant species in the secondary forest and, surprisingly, it shows a 
BENVENUTI-FERREIRA, G. \& COELHO, G.C. 2009. Floristics and structure of the tree component in a Seasonal Forest remnant, Chiapetta, State of Rio Grande do Sul, Brazil. R. Bras. Biosci. 7(4):344-353.

BIODIVERSITAS 2005. Lista da Flora Brasileira ameaçada de extinção segundo avaliação no workshop da Fundação Biodiversitas em 2005. http://www.biodiversitas.org.br/floraBr/lista_florabr.pdf (último acesso em 31/08/2010).

BRASIL. Ministério do Meio Ambiente. 2008. Instrução Normativa ${ }^{\circ}$ 6, de 23 de setembro de 2008. Reconhece Espécies da Flora Ameaçadas de Extinção. Diáro Oficial da República Federativa do Brasil, Brasília, DF. www.icmbio.gov.br/sisbio/legislacao.php?id_arq=42 (último acesso em 14/09/2010).

BROWER, J.E., ZAR, J.H. \& ENDE, C.N. 1998. Field and Laboratory Methods for General Ecology. 4nd ed. McGraw-Hill, Boston.

BUDKE, J. C., ATHAYDE, E.A., GIEHL, E.L.H., ZÁCHIA, R.A. \& EISINGER, S.M. 2005. Composição florística e estratégias de dispersão de espécies lenhosas em uma floresta ribeirinha, arroio Passo das Tropas, Santa Maria, RS, Brasil. Iheringia Sér. Bot. 60(1):17-24.

BUDOWSKI, G. 1965. Distribution of American rain forest species in the light of successional process. Turrialba 15(1):40-42.

BURKART, R. \& FERNÁNDEZ, J.G. 2002. Introducción. In La Selva Misionera - opciones para su conservación y uso sustentable. (R. Burkart, J.P. Cinto, J.C. Chébez, J.G. Fernández \& E. Riegelhaupt, eds.) FUCEMA, Buenos Aires, p. 11-16.

CAMARGO, J.L.C., FERRAZ, I.D.K. \& IMAKAWA, A.M. 2002 Rehabilitation of degraded areas of Central Amazonia using direct sowing of forest tree seeds. Restor. Ecol. 10(4):636-644. http://dx.doi. org/10.1046/j.1526-100X.2002.01044.x

CARVALHO, P.E.R. 1994. Espécies florestais brasileiras: recomendações silviculturais, potencialidades e uso da madeira. EMBRAPA-CNPF, Brasília.

CHAO, A. 1987. Estimating the population size for capture-recapture data with unequal catchability. Biometrics 43(4):783-791. http://dx.doi org/10.2307/2531532

CLARK, J.S., BECKAGE, B., CAMILL, P., CLEVELAND, B., HILLERISLAMBERS, J., LICHTER, J., MCLACHLAN, J., MOHAN, J., \& WYCKOFF, P. 1999. Interpreting recruitment limitation in forests. Amer. J. Bot. 86(1):1-16. http://dx.doi.org/10.2307/2656950

COELHO, G.C. \& MARIATH, J.E.A. 1996. Inflorescences morphology of Ilex L. (Aquifoliaceae) species from Rio Grande do Sul, Brazil. Feddes Repert. 107(1):19-30. http://dx.doi.org/10.1002/fedr.19961070104

COELHO, G.C., RACHWAL, M., SCHNORENBERGER, E., SCHENKEL, E.P. 2000. Efeito do sombreamento sobre a sobrevivência, morfologia e química da erva-mate. In 2nd Congresso Sul-Americano da Erva-mate, 3nd Reunião Técnica da Erva-mate (H. Winge, org.) Encantado, Porto Alegre, p.396-399.

COELHO, G.C., RACHWAL, M.F.G., DEDECEK, R.A., CURCIO, G.R., NIETSCHE, K., SCHENKEL, E.P. 2007. Effect of light intensity on methylxanthine contents of Ilex paraguariensis A. SL. Hil. Biochem. Syst. Ecol. 35(2):75-80. http://dx.doi.org/10.1016/j.bse.2006.09.001

COELHO, G.C. 2009. Interações mutualistas. In Interações Ecológicas e Biodiversidade (M. L. Tissot-Squalli, ed.). 2nd ed. Editora da UNIJUÍ, Ijuí, p.65-91.

COLWELL, R.K. 2006. EstimateS: statistical estimation of species richness and shared species from samples (Software and User's Guide). Version 8. http://viceroy.eeb.uconn.edu/estimates (último acesso em 06/01/2009).

COMPTON, J., HOOKER, T. \& PERAKIS, S. 2007. Ecosystem N distribution and $\delta 15 \mathrm{~N}$ during a century of forest regrowth after agricultural abandonment. Ecosystems 10(7):1197-1208. http://dx.doi.org/10.1007/ s10021-007-9087-y

COTTAM, G. \& CURTIS, J.T. 1956. The use of distance measures in phytosociological sampling. Ecology 37(3):451-460. http://dx.doi. org/10.2307/1930167
DAVIDSON, E.A., DE CARVALho, C. J.R., FIGUEIRA, A.M., ISHIDA, F.Y., OMETTO, J.P.H.B., NARDOTO, G.B., SABÁ, R.T., HAYASHI, S.N., LEAL, E.C., VIEIRA, I.C.G. \& MARTINELLI, L.A. 2007. Recuperation of nitrogen cycling in Amazonian forests following agricultural abandonment. Nature 447(7147):995-999. http://dx.doi. org/10.1038/nature05900

DE FARIA, S.M., LEWIS, G.P., SPRENT, J.I., SUTHERLAND, J.M. 1989. Occurrence of nodulation in the Leguminosae. New Phytol. 111(4):607-619. http://dx.doi.org/10.1111/j.1469-8137.1989.tb02354.x

DIRETORIA DO SERVIÇO GEOGRÁFICO - DSG. 1977. Carta Santo Augusto. Ministério do Exército, Região Sul do Brasil. Carta. Folha SG.22-Y-C-IV-3. Escala 1:50.000.

EPSTEIN, E. \& BLOOM, A.J. 2005. Mineral Nutrition of Plants: Principles and Perspectives. 2nd ed. Sinauer Associates, Sunderland.

FUNDAÇÃO SOS MATA ATLÂNTICA \& INSTITUTO NACIONAL DE PESQUISAS ESPACIAIS - INPE. 2002. Atlas dos remanescentes florestais da Mata Atlântica, período de 1995/2000. Relatório Final. http:// www.sosmataatlantica.org.br (último acesso em 06/11/2008).

GEEVES, G., SEMPLE, B. JOHNSTON, D., JOHNSTON, A., HUGHES, J., KOEN, T. \& YOUNG, J. 2008. Improving the reliability of direct seeding for revegetation in the Central West of New South Wales. Ecol. Manage. Restor. 9(1):68-71. http://dx.doi.org/10.1111/j.1442-8903.2008.00391.x

GEHRING, C., VLEK, P.L.G., DE SOUZA, L.A.G., DENICH, M. 2005. Biological nitrogen fixation in secondary regrowth and mature rainforest of central Amazonia. Agric. Ecosyst. Environ. 111(1-4):237-252. http:// dx.doi.org/10.1016/j.agee.2005.06.009

GIEHL, E.L.H., ATHAYDE, E.A., BUDKE, J.C., GESING, J.P.A., EINSIGER, S.M. \& DO CANTO-DOROW, E.T.S. 2007. Espectro e distribuição vertical das estratégias de dispersão de diásporos do componente arbóreo em uma floresta estacional no sul do Brasil. Acta Bot. Bras. 21(1):137-145. http://dx.doi.org/10.1590/S010233062007000100013

GRANELL-PÉREZ, M.C.G., COELHO, G.C., SILVA, M., SCHMIDT, C., HOLZ, S.M. \& KOCH, S. 1999. Estudio geográfico y botánico de fragmentos forestales en una cuenca del planalto gaucho, Rio Grande do Sul, Brasil. In Voces y territorios de América (F.X. Paunero \& E. Martinelli, eds.). Servei de Publications de La Universitat de Girona, Girona, p.73-80.

HORN, H.S., NATHAN, R. \& KAPLAN, S.R. 2001. Long-distance dispersal of tree seeds by wind. Ecol. Res. 16(5):877-885. http://dx.doi.org/10.1046/ j.1440-1703.2001.00456.x

HOWE, H.F. \& SMALLWOOD, J. 1982. Ecology of seed dispersal. Ann. Rev. Ecol. Syst. 13:201-228. http://dx.doi.org/10.1146/annurev. es.13.110182.001221

HUGHES, R.F., KAUFLSAN, J.B. \& JARAMILLO, V.J. 1999. Biomass, carbon, and nutrient dynamics of secondary forests in a humid tropical region of Mexico. Ecology 80(6):1892-1907.

INTERNATIONAL PLANT NAMES INDEX - IPNI. 2006. The international plant names index database. http://www.ipni.org/index.html (último acesso em 03/12/2010).

JARENKOW, J.A. \& WAECHTER, J.L. 2001. Composição, estrutura e relações florísticas do componente arbóreo de uma floresta estacional no Rio Grande do Sul, Brasil. Rev. Bras. Bot. 24(3):263-272. http://dx.doi. org/10.1590/S0100-84042001000300004

JARENKOW, J.A. \& JURINITZ, C.F. 2003. Estrutura do componente arbóreo de uma floresta estacional na Serra do Sudeste, Rio Grande do Sul, Brasil. Rev. Bras. Bot. 26(4):475-487. http://dx.doi.org/10.1590/ S0100-84042003000400006

JOST, L. 2006. Entropy and diversity. Oikos 113(2):363-375. http://dx.doi. org/10.1111/j.2006.0030-1299.14714.x

KENNARD, D.H. 2002. Secondary forest succession in a tropical dry forest: patterns of development across a 50-year chronosequence in lowland Bolivia. J. Trop. Ecol. 18(1):53-66. http://dx.doi.org/10.1017/ S0266467402002031

MALUF, J.R.T. 2000. Nova classificação climática do Estado do Rio Grande do Sul. Rev. Bras. Agrometeorol. 8(1):141-150. 
MARÍN-SPIOTTA, E., OSTERTAG, R. \& SILVER, W.L. 2007. Long-term patterns in tropical reforestation: plant community composition and aboveground biomass accumulation. Ecol. Appl. 17:828-839. http:// dx.doi.org/10.1890/06-1268

MARONA, H.R.N.; SCHENKEL, E.P.; BERGONCI, J.I. 2003. Phytotoxic activity of Ateleia glazioviana Baill. extracts on lettuce seeds. Acta Farm. Bonaer. 22(1):17-20.

MORELLATO, P.C. 1995. Frutos, frugívoros e a dispersão de sementes. In Ecologia e preservação de uma floresta tropical urbana: Reserva de Santa Genebra (H.F. Leitão Filho \& P.C. Morellato, eds.). Editora da Universidade de Campinas, Campinas, p.64-76.

MORELLATO, L.P.C. \& LEITÃO FILHO, H.F. 1992. Padrões de frutificação e dispersão na serra do Japi. In L.P.C. Morellato (org.). História natural da Serra do Japi: Ecologia e preservação de uma área florestal no sudeste do Brasil. Editora da Universidade de Campinas, Campinas, p.112-141.

NASCIMENTO, A.R.T., LONGHI, S.J., ALVAREZ FILHO, A. \& GOMES, G.S. 2000. Análise da diversidade florística e dos sistemas de dispersão de sementes em um fragmento florestal na região central do Rio Grande do Sul, Brasil. Napaea 12(1):49-67.

NASCIMENTO, A.R.T., LONGHI, S.J. \& BRENA, D.A. 2001. Estrutura e padrões de distribuição espacial de espécies arbóreas em uma amostra de floresta ombrófila mista em Nova Prata, RS. Cienc. Florest. 11(1):105-119.

OLIVEIRA, P.E.A.M. \& MOREIRA, A.G. 1992. Anemocoria em espécies de cerrado e mata de galeria de Brasília, DF. Rev. Bras. Bot. 15(2):163-174.

RAMBO, B.A. 1951. A imigração da selva higrófila no Rio Grande do Sul. A. Bot. Herb. Rodrigues 3:55-91.

RAMBO, B.A. 1956. A fisionomia do Rio Grande do Sul. 2nd ed. Livraria Selbach, Porto Alegre.

REIS, A., BECHARA, F.C., ESPINDOLA, M.B., VIEIRA, N.K. \& SOUZA, L.L. 2003. Restoration of damaged land areas: using nucleation to improve successional processes. Nat. Conserv. 1(1):85-92.

RUCHEL, R.A., NODARI, R.O. \& MOERSCHBACHER, B.M. 2007. Woody plant species richness in the Turvo State park, a large remnant of deciduous Atlantic forest, Brazil. Biodivers. Conserv. 16(6):1699-1714. http://dx.doi.org/10.1007/s10531-006-9044-7

SCHENKEL, V., GASS, S.L.B., LUCCHESE, O.A. \& COELHO, G.C. 2003. Levantamento de cobertura florestal no noroeste rio-grandense: o diagnóstico das APP's a partir de microbacias hidrográficas. In Reflorestamento e Recuperação Ambiental: Biodiversidade e Culturas - a gestão ambiental em foco. (Lucchese, O.A. \& Coelho, G.C., orgs.). Editora da UNIJUÍ, Ijuí, p.192-201.

SOBRAL, M., JARENKOW, J.A, BRACK, P., IRGANG, B., LAROCCA, J. \& RODRIGUES, R.S. 2006. Flora arbórea e arborescente do Rio Grande do Sul, Brasil. Rima/Novo Ambiente, São Carlos.
SOUZA, V.C. \& LORENZI, H. 2008. Botânica Sistemática: guia ilustrado para identificação das famílias de Angiospermas da flora brasileira, baseado em APG II. 2nd ed. Instituto Plantarum, Nova Odessa.

STEVENSON, B.A. \& SMALE, M.C. 2005. Seed bed treatment effects on vegetation and seedling establishment in a New Zealand pasture one year after seeding with native woody species. Ecol. Manage. Restor. 6(2):124-131. http://dx.doi.org/10.1111/j.1442-8903.2005.00229.x

TEDESCO, M.J., GIANELlO, C., BISSANI, C.A., BOHNEN, H. \& VOLKWEISS, S.J. 1995. Análise de solo, plantas e outros materiais. Departamento de Solos/UFRGS, Porto Alegre.

UHL, C., BUSCHBACHER, R. \& SERRÃO, E. A. S. 1988. Abandoned pastures in Eastern Amazonia. I. Patterns of plant succession. J. Ecol. 76(3):663-681. http://dx.doi.org/10.2307/2260566

UNITED STATES DEPARTAMENT OF AGRICULTURE - USDA. 1999. Soil taxonomy - a basic system of soil classification for making and interpreting soil surveys. 2nd ed. U.S. Government Printing Office, Washington.

VAN DER PIJL, L. 1982. Principles of dispersal in higher plants. Springer-Verlag, Berlim.

VASCONCELLOS, J.M.O., DIAS, L.L., SILVA, C.P., SOBRAL, M. 1992. Levantamento florístico de uma área de mata subtropical no Parque Estadual do Turvo, Tenente Portela, RS. Rev. Inst. Florest. 4:252-259.

VITOUSEK, P. M., MATSON, P. A. \& VAN CLEVE, K. 1989. Nitrogen availability and nitrification during succession: primary, secondary, and old-field seres. Plant Soil 115(2):229-239. http://dx.doi.org/10.1007/ BF02202591

WHITE, E., TUCKER, N., MEYERS, N., WILSON, J. 2004. Seed dispersal to revegetated isolated rainforest patches in North Queensland. For. Ecol. Manage. 192(2-3):409-426. http://dx.doi.org/10.1016/j. foreco.2004.02.002

WILLS, C., CONDIT, R., FOSTER, R.B., \& HUBBELL, S.P. 1997. Strong density- and diversity-related effects help to maintain tree species diversity in a neotropical forest. Proc. Natl. Acad. Sci. USA 94(4):1252-1257. http://dx.doi.org/10.1073/pnas.94.4.1252

WILLS, C., HARMS, K.E., CONDIT, R., KING, D., THOMPSON, J., HE, F., MULLER-LANDAU, H.C., ASHTON, P., LOSOS, E., COMITA, L., HUBBELL, S., LAFRANKIE, J., BUNYAVEJCHEWIN, S., DATTARAJA, H. S., DAVIES, S., ESUFALI, S., FOSTER, R., GUNATILLEKE, N., GUNATILLEKE, S., HALL, P., ITOH, A., JOHN, R., KIRATIPRAYOON, S., DE LAO, S.L., MASSA, M., NATH, C., NOOR, M.N.S., KASSIM, A.R., SUKUMAR, R., SURESH, H.S., SUN, I-F., TAN, S., YAMAKURA, T. \& ZIMMERMAN, J. 2006. Nonrandom processes maintain diversity in tropical forests. Science 311(5760): 527-531. http://dx.doi.org/10.1126/ science. 1117715

ZANINI, L. \& GANADE, G. 2005. Restoration of Araucaria Forest: the role of perches, pioneer vegetation, and soil fertility. Restor. Ecol. 13(3):507514. http://dx.doi.org/10.1111/j.1526-100X.2005.00063.x 